\title{
PRODUCCIÓN MASIVA DE Ceraeochrysa valida (BANKS) (NEUROPTERA: CHRYSOPIDAE) A $30^{\circ} \mathrm{C}$
}

\section{MASS PRODUCTION OF Ceraeochrysa valida (BANKS) (NEUROPTERA: CHRYSOPIDAE) AT $30^{\circ} \mathrm{C}$}

\author{
Martín Palomares-Pérez ${ }^{1 *}$, Mónica Isabel Barajas-Romero ${ }^{1}$, y Hugo Cesar Arredondo-Bernal ${ }^{1}$ \\ ${ }^{1}$ Centro Nacional de Referencia de Control Biológico, SENASICA-DGSV Km 1.5 Carretera Tecomán- \\ Estación FFCC, Colonia Tepeyac, Colima, México. C.P. 28110; \\ * Autor para correspondencia E-mail: mpalomares@colpos.mx
}

\section{RESUMEN}

Actualmente, el área de insectos entomófagos del Centro Nacional de Referencia de Control Biológico (CNRCB) en Tecomán, Colima, México, se encuentra generando y validando alternativas que involucran la producción masiva de Ceraeochrysa valida. Este insecto es un depredador generalista, cuya distribución en diferentes estados de la República hacen inferir que posee gran potencial para ser considerado en programas de control biológico. El CNRCB ha identificado que la producción masiva de este depredador requiere ahorrar tiempo y alimento en condiciones artificiales de reproducción. De acuerdo a esto, el presente trabajo tiene como objetivo evaluar la producción de huevos de $C$. valida a una temperatura de $30^{\circ} \mathrm{C}$ con la finalidad de acortar el ciclo biológico y producir una mayor cantidad de ejemplares en el menor tiempo posible. Los resultados indican que el ciclo biológico redujo su longevidad 9,33 \pm 1,1 días comparado con el testigo. La larva II registró la mayor reducción $(2,33 \pm 0,21)$. La producción de huevos fue de 5561,22 huevos a $30^{\circ} \mathrm{C}$ y 4924,82 del testigo, las pruebas estadísticas de Wilcoxon $(\operatorname{Pr}>z=0,3106 ; \operatorname{Pr}>|z|=0,6212)$, Kruskall-Wallis $(\operatorname{Pr}>$ chi-cuadrado $=0,6171)$ y prueba de $t(\operatorname{Pr}>z=0,3114 ; \operatorname{Pr}>|z|=0,6228)$ indican que no hay diferencia significativa con el testigo. Los resultados obtenidos indican que es factible producir masivamente $C$. valida a $30^{\circ} \mathrm{C}$, ahorrando tiempo y alimento en condiciones artificiales de producción.

Palabras clave: control biológico, depredador, temperatura, producción masiva.

\section{ABSTRACT}

At present, the entomophagous insect area of the National Biological Control Reference Center (CNRCB) in Tecomán, Colima, Mexico, is generating and validating alternatives that involve the mass production of Ceraeochrysa valida. This insect is a generalist predator widely distributed in different states of the Republic, and therefore has a great potential in biological control programs. The CNRCB has identified that the massive production of this predator requires saving time and food under artificial breeding conditions. Therefore, the objective of this study is to evaluate the production of eggs of $C$. valida at a temperature of $30^{\circ} \mathrm{C}$ in order to shorten the biological cycle and produce a larger number of specimens in the shortest time possible. The results indicate that the biological cycle reduced its longevity $9.33 \pm 1.1$ days compared to the control. Larva II recorded the greatest reduction $(2.33 \pm 0.21)$. Egg production was 5561.22 eggs at $30^{\circ} \mathrm{C}$, and 4924.82 eggs in the control. Wilcoxon's statistical tests $(\operatorname{Pr}>\mathrm{z}=0.3106$; $\operatorname{Pr}>\mathrm{z}=0.6212)$, Kruskall-Wallis $(\operatorname{Pr}>$ chi-square $=$ 0.6171) and $t$-test $(\operatorname{Pr}>z=0.3114 ; \operatorname{Pr}>|z|=0.6228)$ show no significant difference compared to the 
control. The results obtained indicate that the massive production of $C$. valida is possible at $30^{\circ} \mathrm{C}$, saving time and food under artificial breeding conditions.

Key words: biological control, predator, temperature, mass production.

\section{INTRODUCCIÓN}

Las especies de la familia Chrysopidae son los insectos más abundantes del orden Neuroptera (Penny, 2002). Brooks y Barnard (1990) citaron cerca de 1200 especies y subespecies reconocidas, clasificadas en 86 géneros y subgéneros. Está considerada como una de las familias de entomófagos más importantes del orden Neuroptera, debido a que 15 géneros presentan especies con potencial como agentes de control biológico (New, 2001). Las larvas de todas las especies y los adultos de algunos géneros son depredadores y se alimentan de una amplia variedad de insectos fitófagos tales como áfidos (Hemiptera: Aphididae), cóccidos (Hemiptera: Coccidae), mosquitas blancas (Hemiptera: Aleyrodidae) y otros insectos de cuerpo blando que se localizan en el follaje. Por esta razón, algunas especies se reproducen actualmente de manera masiva y se utilizan exitosamente para el control biológico de plagas agrícolas (Penny, 2002).

Dentro de la familia Chrysopidae se encuentra Ceraeochrysa valida (Banks) (Neuroptera: Chrysopidae), considerado un depredador generalista (Winterton y Makarkin, 2010) y cuya distribución en diferentes estados de la república mexicana (Valencia et al., 2006; CortezMondaca et al., 2011; Córdoba-Urtiz et al., 2014) hacen suponer que tiene gran potencial para ser incluido en programas de control biológico (Palomares-Pérez et al., 2016), ya sea por aumento o conservación. Estudios de laboratorio indican que es capaz de alimentarse hasta de 100 ninfas de Diaphorina citri Kuwayama 1908, (Hemiptera: Liviidae) en 24 h (Cortez-Mondaca et al., 2011), plaga considerada como la más importante de los cítricos debido a que transmite la bacteria Candidatus Liberibacter sp. (Martínez y Wallace, 1967; Bové, 2006) responsable de la enfermedad Huanglongbing (HLB) (Huang et al., 1984; Bové, 2006) y causante de la muerte de millones de árboles en el mundo (Halbert y Manjunath, 2004; Bové, 2006).

Actualmente, el área de Insectos Entomófagos del Centro Nacional de Referencia de Control Biológico (IE-CNRCB) se encuentra desarrollando y validando alternativas que involucran la producción y uso de C. valida como uno de los depredadores con gran potencial para ser utilizado en el control biológico de diversas plagas agrícolas (Tauber et al., 2000).
De todos los factores ambientales, el que ejerce un efecto mayor sobre el desarrollo de los insectos es probablemente, la temperatura. Ello se debe principalmente a su importante incidencia sobre los procesos bioquímicos (Wagner et al., 1984). Por lo tanto, no es extraño utilizar la temperatura y su influencia sobre el tiempo de desarrollo de los insectos. De acuerdo a esto, el presente trabajo tuvo como objetivo evaluar la influencia de la temperatura a $30^{\circ} \mathrm{C}$ en el ciclo biológico y producción masiva de huevos de $C$. valida, con la finalidad de acortar el ciclo y producir mayor cantidad de individuos en el menor tiempo posible.

\section{MATERIALES Y MÉTODOS}

Se utilizaron cámaras bioclimáticas controladas a $30^{\circ} \mathrm{C} \pm 1$, manteniendo condiciones fijas de 60 a $65 \%$ HR y 14:10 (L:O). La temperatura de $25^{\circ} \mathrm{C}$ utilizada en la producción de crisopidos del laboratorio de IE-CNRCB se consideró como testigo.

El trabajo se realizó en el laboratorio de IE-CNRCB, ubicado en la ciudad de Tecomán, Coli-

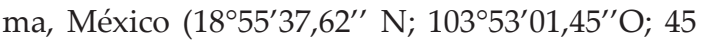
m.s.n.m.).

Ciclo de vida: Con un microscopio estereoscópico se seleccionaron del pie de cría del laboratorio de IE-CNRCB 100 huevos de C. valida con $24 \mathrm{~h}$ de edad. Se colocaron con un pincel de cerdas finas en cajas de Petri de plástico de $5 \mathrm{~cm}$ de diámetro. Los huevos se observaron diariamente y una vez que eclosionaron a larva I se registró y se estableció como la duración del periodo de incubación.

Para comprobar el periodo larval, las larvas recién emergidas se tranfirieron individualmente a cajas de Petri de $5 \mathrm{~cm}$ de diámetro y se alimentaron hasta completar su ciclo con huevos de Sitotroga cerealella (Oliver) (Lepidoptera: Gelechiidae). La duración de cada ínstar se registró mediante el tamaño de la larva y la presencia de la exuvia.

El estado de pupa se estableció cuando se observó la presencia del cocón y culminó con la emergencia del adulto.

Una vez que emergió el adulto, este se trasladó y se observó hasta su muerte en vasos de unicel de $8 \mathrm{~cm}$ de diámetro por $6 \mathrm{~cm}$ de alto. Para facilitar la aireación y evitar la fuga del insecto, en la tapa superior se hizo una abertura de $4 \mathrm{~cm}$ de diámetro que se cubrió con tela de organza. 
Además en la parte lateral inferior se realizó una perforación de $1,5 \mathrm{~cm}$ de diámetro, la cual se tapó con algodón y se humedeció diariamente para suministrar agua al adulto. En el interior del vaso se colocó un trozo de papel bond de $1,5 \times 2 \mathrm{~cm}$ impregnado con dieta artificial (miel, levadura de cerveza, polen, ácido ascórbico y espirulina) como alimento.

El análisis de la duración del ciclo biológico se estimó con la comparación de la media y error estándar (Ott, 1993) del testigo.

Producción de huevos: Se utilizaron cilindros de PVC de $24,5 \mathrm{~cm}$ de diámetro por $30 \mathrm{~cm}$ de alto. Se cubrieron con tela mosquitera (Tela mosquitera de plástico verde en bobina surtek Hm4) en ambos extremos y se colocaron a razón 1:1 (: $\left.0^{\wedge}\right) 200$ adultos de la misma edad de $C$. valida: Los adultos se alimentaron cada tercer día con la dieta artificial antes mencionada colocada a lo largo de una regla de plástico de $30 \mathrm{~cm}$ de largo por $3 \mathrm{~cm}$ de ancho y ubicada de forma inclinada en el interior de los cilindros. Para abastecer de agua a los insectos, en la parte superior externa se colocó diariamente algodón húmedo.

Como sustrato de oviposición se forró el interior de los cilindros con cartulina negra de $80 \mathrm{~cm}$ de largo por $30 \mathrm{~cm}$ de alto, la cual se retiraba y sustituía diariamente por uno nuevo. Con la ayuda de tijeras de punta fina, los huevos colocados en la cartulina se recortaron por el pedicelo.

El registro de la cantidad de huevos se realizó pesándolos en forma conjunta en una báscula de precisión (Precisa Gravimetric AG Dietikon, Switzerlan XT220A). La equivalencia de huevos por gramo se registró mediante el conteo del número de huevos en un gramo (3600 huevos $\mathrm{g}^{-1}$ ).

El análisis estadístico para comparar la producción de huevos se realizó con una prueba t-student y al no ajustarse los datos a los supuestos de normalidad, estos fueron sometidos a una prueba no paramétrica utilizando el programa estadístico SAS 9.2 (2008).

\section{RESULTADOS Y DISCUSIÓN}

La duración del ciclo biológico de huevo hasta la emergencia del adulto a $30^{\circ} \mathrm{C}$ fue de $26,3 \pm 0,5$ días, con un rango de 25 a 29 días. Del total de individuos evaluados, 76 insectos alcanzaron el estado adulto; 41 fueron hembras y 35 machos, cuyo ciclo biológico se completó a los 27,9 y 24,6 días, respectivamente. El huevo eclosionó en promedio en $4,1 \pm 0,06$ días, el estado larval tuvo en promedio una duración de 11,6 6 0,34 días, donde LIII con 4,5 \pm 0,12 días es el instar más prolongado. La pupa por su parte presentó una longevidad de 10,6 6 0,18 días.
El testigo completó su ciclo biológico a los $36,2 \pm 01,68$ días, con un rango de 34 a 41. Se registraron 68 insectos que completaron su ciclo hasta la emergencia del adulto; de los cuales 30 fueron hembras y 38 machos, y su ciclo biológico comprendió 36,6 y 35,7 días, respectivamente. El huevo eclosionó en promedio a los 5,6 $\pm 0,13$ días, y el instar larval que duró más tiempo fue larva III con 5,13 $\pm 0,048$ días. El estado de pupa duró $14,26 \pm 0,43$ días.

En comparación con el testigo, el análisis de las medias indica que el ciclo biológico de $C$. valida a $30^{\circ} \mathrm{C}$ redujo su longevidad $9,93 \pm 1,1$ días de huevo a la emergencia del adulto. La larva II con 2,3 $\pm 0,21$ días es la de mayor reducción. En condiciones controladas de $24 \pm 2{ }^{\circ} \mathrm{C}$, HR de 60 $65 \%$ y alimentado con ninfas de $D$. citri, PachecoRueda et al. (2013) obtuvieron un ciclo biológico de $C$. valida de huevo a adulto de 35 días, dato que coincide con el testigo pero que difiere con el tratamiento a $30^{\circ} \mathrm{C}$.

En estudios realizados con otra especie de Crysopidos, Khan et al. (2012) y Nadeen et al. (2012) indican que la tasa de desarrollo de Chrysoperla carnea (Stephens) disminuye gradualmente con el aumento de la temperatura. Autores como Venzon y Carvalho (1993) observaron que Ceraeochrysa cubana (Hagen) (Neuroptera: Chrysopidae) decrece su desarrollo larval cuando se eleva la temperatura.

Producción de huevos. La producción de huevos no se ajustó a los supuestos de normalidad como lo expresa el estadístico KolmogorovSmirnov ( $\mathrm{p}<0,0357$ ), por lo tanto estos se sometieron a una prueba de tendencia central no paramétrica de Wilcoxon-Mann-Whitney (Siegel y Castellan, 1995). El paquete estadístico SAS detectó que la variable temperatura tiene dos niveles, por lo que se realizaron tres pruebas: prueba de Wilconxon $(\operatorname{Pr}>\mathrm{z}=0,3106$; $\operatorname{Pr}>|\mathrm{z}|=$ 0,6212), prueba $t$ aproximada $(\operatorname{Pr}>z=0,3114$; $\operatorname{Pr}$ $>|z|=0,6228)$ y prueba de Kruskall-Wallis (Pr $>$ chi-cuadrado $=0,6171)$. Estas pruebas indicaron que no existe diferencia estadística entre la población a $30^{\circ} \mathrm{C}$ con una producción de huevo de 1,54 $\pm 0,02$ gr (5561,22 huevos) y el testigo con $1,36 \pm 0,01$ gr (4924,82 huevos).

En la Fig. 1 se observa que en los primeros 20 días, la producción de huevos a $30^{\circ} \mathrm{C}$ es superior numericamente al testigo, dato que indica que al aumentar la temperatura, aumentó la oviposición de 200 individuos. Esto se debe a que el periodo de preoviposición se acortó de 1 a 2 días.

Con un total de 39,93 \pm 4,33 días para la población a $30^{\circ} \mathrm{C}$ y $47,80 \pm 4,29$ para el testigo, la prueba de $t$ con un $\alpha=0,05$ indica que no existe diferencia estadística en la longevidad entre ambas poblaciones ( $\mathrm{t}$-value $=0,16 ; \operatorname{Pr}>0,8781$ ). 


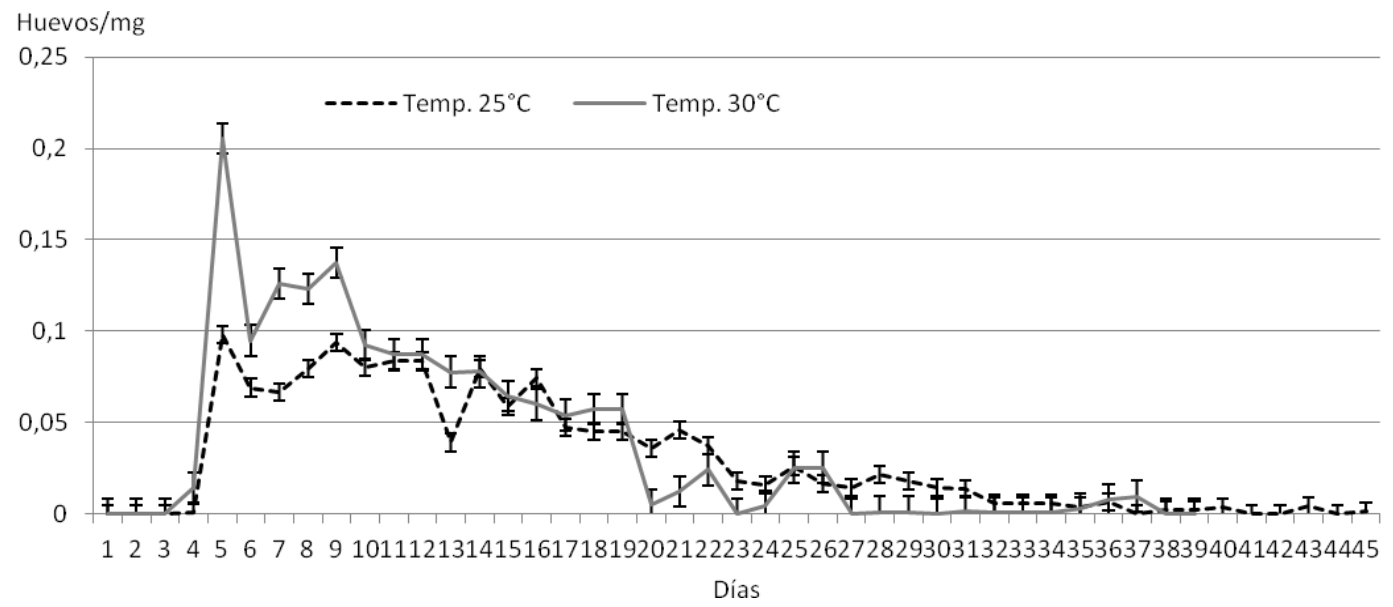

Fig. 1. Producción de huevo $(\mathrm{mg})$ de C. valida a $25^{\circ} \mathrm{C}$ y $30^{\circ} \mathrm{C}$ en el laboratorio de entomófagos del Centro Nacional de Referencia de Control Biológico (CNRCB).

Fig. 1. Egg production (mg) of $C$. validated at $25^{\circ} \mathrm{C}$ and $30^{\circ} \mathrm{C}$ in the entomophagous laboratory of the Centro Nacional de Referencia de Control Biológico (CNRCB).

\section{CONCLUSIÓN}

Los resultados de la presente investigación permiten concluir que es factible producir masivamente $\mathrm{C}$. valida a $30^{\circ} \mathrm{C}$, ahorrando tiempo, alimento y condiciones artificiales de producción.

\section{LITERATURA CITADA}

Brooks, S.J., and P.C. Barnard. 1990. The green lacewings of the world: a generic review (Neuroptera: Chrysopidae). Bulletin of the British Museum Natural History (Entomology) 59:117-286.

Bové, J.M. 2006. Huanglongbing: A destructive, newly emerging, century-old disease of citrus. Journal of Plant Pathology 88:7-37.

Córdoba-Urtiz, E.G., M. Palomares-Pérez, J.A. Sánchez-González, G. Moreno-Carrillo, y H.C. Arredondo-Bernal. 2014. Avances en el estudio de diversidad de especies de la familia Chrysopidae (Neuroptera) en la zona citrícola del estado de Colima. En Lozano-Contreras, M.G., M.J. BurgosTan, y J.I. López-Arroyo (eds.). Memoria: XXXVII Congreso Nacional de Control Biológico, Mérida, Yucatán, México. 450 p. 6 y 7 de noviembre, 2014. Centro Regional de Investigación del Sureste (CIRSE), Campo Experimental Mocochá. CD-ROM. Memoria Científica. México.
Cortez-Mondaca, E., J.I. López-Arroyo, L. Rodríguez-Ruíz, M.P. Partida-Valenzuela, J. Pérez-Márquez, y V.M. González-Calderón. 2011. Capacidad de depredación de especies de chrysopidae asociadas a Diaphorina citri Kuwayama en los cítricos de Sinaloa, México. En $2^{\circ}$ Simposio Nacional sobre investigación para el manejo del Psílido Asiático de los Cítricos y el Huanglongbing en México. 5-6 diciembre 2011. INIFAP, Montecillo, Texcoco, México.

Halbert, S.E, y K.L. Manjunath. 2004. Asian citrus psyllids (Sternorrhyncha: Psyllidae) and greening disease of citrus: a literature review and assessment of risk in Florida. Florida Entomologist 87(3):330-353.

Huang, C.H., M.Y. Tsai, and C.L. Wang. 1984. Transmission of citrus likubin by a psyllid, Diaphorina citri. Journal Agricultural Research China 33(1):15-72.

Khan,J., E.Ul-Haq, N.Akhtar,W.A.Gillani, N.Assad, M.A. Masood, et al. 2012. Effect of temperature on biological parameters of immature stages of Chrysoperla carnea (Neuroptera: Chrysopidae) feeding on rice meal moth, Corcyra cephalonica eggs. Journal of Agricultural Research Pakistan 25(3):224-227.

Martinez, A.L., and J.M. Wallace. 1967. Citrus leafmottle-yellows disease in the Philippines and transmission of the causal virus by a psyllid, Diaphorina citri. In Donovan, N.J., G.A.CBeattie, G.A. Chambers, P. Holford, A. Englezou, S. Hardy, et al. 2012. First report of 'Candidatus Liberibacter asiaticus' in Diaphorina communis. Australasian Plant Disease Notes 7:1-4. 
Nadeem, S., M. Hamed, M.K. Nadeem, M., Hasnain, B.M. Atta, N.A. Seeed, et al. 2012. Comparative study of developmental and reproductive characteristics of Chrysoperla carnea (Stephens) (Neuroptera: Chrysopidae) at different rearing temperatures. The Journal of Animal \& Plant Sciences 22(2):399402.

New, T.R. 2001. Introduction to the systematics and distribution of Coniopterygidae, Hemerobiidae, and Chrysopidae used in pest management. p. 6-28. In P. McEwen, T.R. New, and A.E. Whittington (eds.) Lacewings in the Crop Environment. Cambridge University, Cambridge, England.

Ott, R.L. 1993. An introduction to stadistical methods and data analysis. p. 354-418. 4th ed. Duxbury Press, Belmont, California, USA.

Pacheco-Rueda, I., J.R. Lomeli-Flores, J.I. LópezArroyo, H. González-Hernández, and J. Romero-Napoles. 2013. Biology and fecundity of Ceraeochrysa valida (Neuroptera: Chrysopidae) reared on Diaphorina citri Kuwayama (Hemiptera: Psyllidae). Cartel (D0456). 12 de noviembre de 2013. Centro de Convenciones de Austin, Texas, USA. Available at https://esa.confex.com/esa/2013/ webprogram/Session20618.html. (Accessed 3 November 2016).

Palomares-Pérez, M., M.A. Ayala-Zermeño, B. Rodrígez-Velez, J.J. De La Cruz-Llanas, J.A. Sánchez-González, H.C. Arredondo-Bernal y E.G. Córdoba-Urtiz. 2016. Abundancia y depredación de Ceraeochrysa valida (Neuroptera: Chrysopidae) sobre Diaphorina citri (Hemiptera: Liviidae) en Colima, México. Chilean Journal of Agricultural \& Animal Sciences, ex Agro-Ciencia 32(3):234243.

Penny, N.D. 2002. A guide to the lacewings (Neuroptera) of Costa Rica. Proceedings of the California Academy of Sciences 53(12):161-457.
SAS Institute. 2008. SAS Users Guide: Statistics version 9.02 for Windows. SAS Institute Inc., Cary, North Carolina, USA.

Siegel, S., y N.J. Castellan. 1995. Estadística no Paramétrica Aplicada a las Ciencias de la Conducta. 437 p. Editorial Trillas, México.

Tauber, C.A., T. De León, N.D. Penny, M.J. Tauber. 2000. The genus Ceraeochrysa (Neuroptera: Chrysopidae) of America north of Mexico: larvae, adults, and comparative biology. Annals of the Entomological Society of America 93(6):1195-1221.

Valencia-Luna, L.A., J. Romero-Nápoles, J. Valdez-Carrasco, J.L. Carrillo-Sánchez, y V. López-Martínez. 2006. Taxonomía y registro de Chrysopidae (Insecta: Neuroptera) en el Estado de Morelos, México. Acta Zoológica Mexicana (nueva serie) 22(1):17-61.

Venzon, M., and C.F. Carvalho. 1993. Desenvolvimento larval, pré-pupal e pupal de Eraeochrysa cubana (Hagen) (Neuroptera: Chrysopidae) em diferentes dietas e temperaturas. Anais da Sociedade Entomológica do Brasil, Londrina 22:477483.

Wagner, T.L., H. Wu, P.J.H. Sharpe, R.M. Schoolfield, and R.N. Coulson. 1984. Modeling insect development rates: a literature review and application of a biophysical model. Annals of the Entomological Society of America 77:208225.

Winterton, S.L., and V.N. Makarkin. 2010. Phylogeny of moth lacewings and giant lacewings (Neuroptera: Ithonidae, Polystoechotidae) using DNA sequence data, morphology and fossils. Annals of the Entomological Society of America 103:511522. 